\title{
Simulations of Aging and Plastic Deformation in Polymer Glasses
}

\author{
Mya Warren* and Jörg Rottler \\ Department of Physics and Astronomy, The University of British Columbia, \\ 6224 Agricultural Road, Vancouver, BC, V6T 1Z1, Canada
}

(Dated: August 14, 2018)

\begin{abstract}
We study the effect of physical aging on the mechanical properties of a model polymer glass using molecular dynamics simulations. The creep compliance is determined simultaneously with the structural relaxation under a constant uniaxial load below yield at constant temperature. The model successfully captures universal features found experimentally in polymer glasses, including signatures of mechanical rejuvenation. We analyze microscopic relaxation timescales and show that they exhibit the same aging characteristics as the macroscopic creep compliance. In addition, our model indicates that the entire distribution of relaxation times scales identically with age. Despite large changes in mobility, we observe comparatively little structural change except for a weak logarithmic increase in the degree of short-range order that may be correlated to an observed decrease in aging with increasing load.
\end{abstract}

PACS numbers: 81.40.Lm, 81.40.Lg, 83.10.Rs

\section{INTRODUCTION}

Glassy materials are unable to reach equilibrium over typical experimental timescales [1, 2, 3]. Instead, the presence of disorder at temperatures below the glass transition permits only a slow exploration of the configurational degrees of freedom. The resulting structural relaxation, also known as physical aging [4], is one of the hallmarks of glassy dynamics and leads to material properties that depend on the wait time $t_{w}$ since the glass was formed. While thermodynamic variables such as energy and density typically evolve only logarithmically, the relaxation times grow much more rapidly with wait time $[3,4,[5]$.

Aging is a process observed in many different glassy systems, including colloidal glasses [6], microgel pastes [7], and spin glasses [8], but is most frequently studied in polymers due to their good glass-forming ability and ubiquitous use in structural applications. Of particular interest is therefore to understand the effect of aging on their mechanical response during plastic deformation [5]. In a classic series of experiments, Struik [4] studied many different polymer glasses and determined that their stiffness universally increases with wait time. However, it has also been found that large mechanical stimuli can alter the intrinsic aging dynamics of a glass. Cases of both decreased aging (rejuvenation) [4] and increased aging (overaging) [9, 10] have been observed, but the interpretation of these findings in terms of the structural evolution remains controversial [11, 12].

The formulation of a comprehensive molecular model of the non-equilibrium dynamics of glasses has been impeded by the fact that minimal structural change occurs during aging. Traditional interpretations of aging presume that structural relaxation is accompanied by a

*Electronic address: mya@phas.ubc.ca decrease in free volume available to molecules and an associated reduction in molecular mobility [4]. While this idea is intuitive, it suffers from several limitations. First, the free volume has been notoriously difficult to define experimentally. Also, this model does not seem compatible with the observed aging in glassy solids under constant volume conditions [13], and cannot predict the aging behavior under complex thermo-mechanical histories. Modern energy landscape theories describe the aging process as a series of hops between progressively deeper traps in configuration space [14, 15]. These models have had some success in capturing experimental trends, but have yet to directly establish a connection between macroscopic material response and the underlying molecular level processes. Recent efforts to formulate a molecular theory of aging are promising but require knowledge of how local density fluctuations control the relaxation times in the glass [16].

Molecular simulations using relatively simple models of glass forming solids, such as the binary Lennard-Jones glass [17] or the bead spring model [18] for polymers, have shown rich aging phenomenology. For instance, calculations of particle correlation functions have shown explicitly that the characteristic time scale for particle relaxations increases with wait time [19]. Recent work $[13,20]$ has focused on the effect of aging on the mechanical properties; results showed that the shear yield stress (defined as the overshoot or maximum of the stress-strain curve) in deformation at constant strain rate generally increases logarithmically with $t_{w}$. Based on a large number of simulations at different strain rates and temperatures, a phenomenological rate-state model was developed that describes the combined effect of rate and age on the shear yield stress for many temperatures below the glass transition [21].

In contrast to the strain-controlled studies described above, experiments on aging typically impose a small, constant stress and measure the resulting creep as a function of time and $t_{w}[4]$. In this study, we perform molecu- 
lar dynamics simulations on a coarse grained, glass forming polymer model in order to investigate the relationship between macroscopic creep response and microscopic structure and dynamics. In Section ПII we determine creep compliance curves for different temperatures and applied loads (in the sub-yield regime) and find that, as in experiments, curves for different ages can be superimposed by rescaling time. The associated shift factors exhibit a power-law dependence on the wait time, and the effect of aging can be captured by an effective time as originally envisioned by Struik [4]. In Section [IB, we compute microscopic mobilities and the full spectrum of relaxation times and show their relationship to the creep response. Additionally, we study several parameters that are sensitive to the degree of short-range order in Section IIIC We detect very little evolution toward increased local order in our polymer model, indicating that short range order is not a sensitive measure of the mechanical relaxation times responsible for the creep compliance of glassy polymers.

\section{SIMULATIONS}

We perform molecular dynamics (MD) simulations with a well-known model polymer glass on the beadspring level. The beads interact via a non-specific van der Waals interaction given by a 6-12 Lennard-Jones potential, and the covalent bonds are modeled with a stiff spring that prevents chain crossing [22]. This level of modeling does not include chemical specificity, but allows us to study longer aging times than a fully atomistic model and seems appropriate to examine a universal phenomenon found in all glassy polymers. All results will be given in units of the diameter $a$ of the bead, the mass $m$, and the Lennard-Jones energy scale, $u_{0}$. The characteristic timescale is therefore $\tau_{L J}=\sqrt{m a^{2} / u_{0}}$, and the pressure and stress are in units of $u_{0} / a^{3}$. The Lennard-Jones interaction is truncated at $1.5 a$ and adjusted vertically for continuity. All polymers have a length of 100 beads, and unless otherwise noted, we analyze 870 polymers in a periodic simulation box. Results are obtained either with one large simulation containing the full number of polymers, or with several smaller simulations, each starting from a unique configuration, whose results are averaged. The large simulations and the averaged small simulations provide identical results. The small simulations are used to estimate uncertainties caused by the finite size of the simulation volume.

To create the glass, we begin with a random distribution of chains and relax in an ensemble at constant volume and at a melt temperature of $1.2 u_{0} / k_{B}$. Once the system is fully equilibrated, it is cooled over $750 \tau_{L J}$ to a temperature below the glass transition at $T_{g} \approx 0.35 u_{0} / k_{B}[18]$. The density of the melt is chosen such that after cooling the pressure is zero. We then switch to an NPT ensemble - the pressure and temperature are controlled via a Nosé-Hoover thermo-
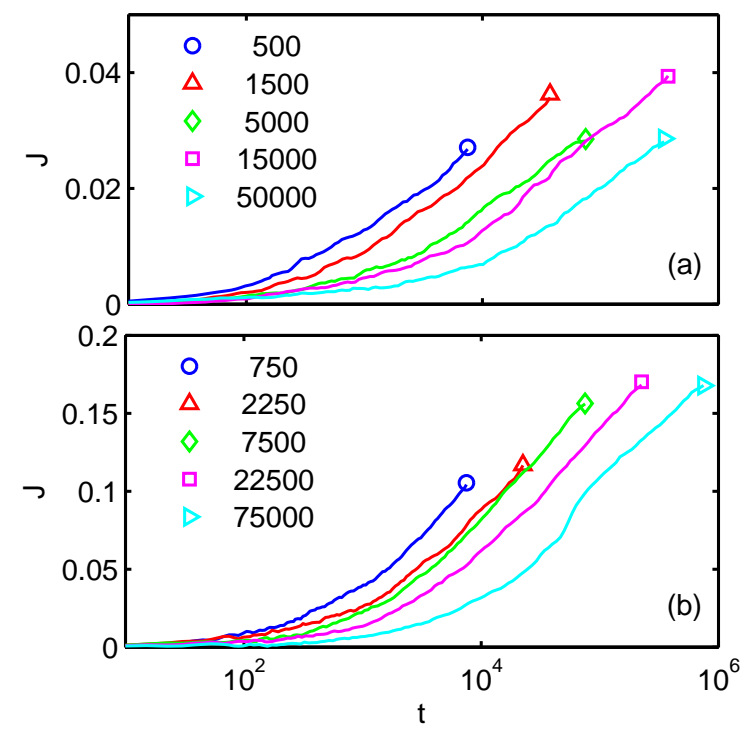

FIG. 1: Simulated creep compliance $J\left(t, t_{w}\right)$ at a glassy temperature of $T=0.2 u_{0} / k_{B}$ for various wait times $t_{w}$ (indicated in the legend in units of $\tau_{L J}$ ). A uniaxial load of (a) $\sigma=0.4 u_{0} / a^{3}$ and (b) $\sigma=0.5 u_{0} / a^{3}$ is applied to the aged glasses. The strain during creep is monitored over time to give the creep compliance.

stat/barostat - with zero pressure and age for various wait times $\left(t_{w}\right)$ between 500 to $75,000 \tau_{L J}$. The aged samples undergo a computer creep experiment where a uniaxial tensile stress (in the z-direction) is ramped up quickly over $75 \tau_{L J}$, and then held constant at a value of $\sigma$, while the strain $\epsilon=\Delta L_{z} / L_{z}$ is monitored. After an initial elastic deformation, the glass slowly elongates in the direction of applied stress due to structural relaxations. In the two directions perpendicular to the applied stress, the pressure is maintained at zero.

\section{RESULTS}

\section{A. Macroscopic Mechanical Deformation}

Historically, measurements of the creep compliance have been instrumental in probing the relaxation dynamics of glasses, and continue to be the preferred tool in investigating the aging of glassy polymers [15, 23, 24]. In his seminal work on aging in polymer glasses, Struik [4] performed an exhaustive set of creep experiments on different materials, varying the temperature and the applied load. In this section, we perform a similar set of experiments with our model polymer glass. The macroscopic creep compliance is defined as

$$
J\left(t, t_{w}\right)=\frac{\epsilon\left(t, t_{w}\right)}{\sigma} .
$$






FIG. 2: The same data as Fig. 1 is shown with the curves shifted by $a_{J}\left(t_{w}\right)$ to form a master curve. The dashed lines are fits to the master curves using the effective time formulation, and the dotted line is a short-time fit for comparison (see text).

Compliance curves $J\left(t, t_{w}\right)$ for several temperatures and stresses were obtained as a function of wait time since the quench; representative data is shown in Figure 1 . The curves for different wait times appear similar and agree qualitatively with experiment. An initially rapid rise in compliance crosses over into a slower, logarithmic increase at long times. The crossover between the two regimes increases with increasing wait time. Struik showed that experimental creep compliance curves for different ages can be superimposed by rescaling the time variable by a shift factor, $a_{J}$,

$$
J\left(t, t_{w}\right)=J\left(t a_{J}, t_{w}^{\prime}\right) .
$$

This result is called time-aging time superposition [4, 5]. Simulated creep compliance curves from Fig. 1 can similarly be superimposed, and the resulting master curve is shown in Fig. 2 .

Shift factors required for this data collapse are plotted versus the wait time in Fig. 3, All data fall along a straight line in the double-logarithmic plot, clearly indicating power law behavior:

$$
a_{J} \sim t_{w}^{-\mu} .
$$

This power law in the shift factor is characteristic of aging. $\mu$ is called the aging exponent, and has been found experimentally to be close to unity for a wide variety of glasses in a temperature range near $T_{g}[4]$.

Figure 4 shows the effect of stress and temperature on the aging exponent, as determined from linear fits to the data in Fig. 3. At $T=0.2 u_{0} / k_{B}, \mu$ is close to one for small stresses, but decreases strongly with stress. This apparent erasure of aging by large mechanical deformations has been called "mechanical rejuvenation" [25]. Experiments have frequently found a stress dependence of
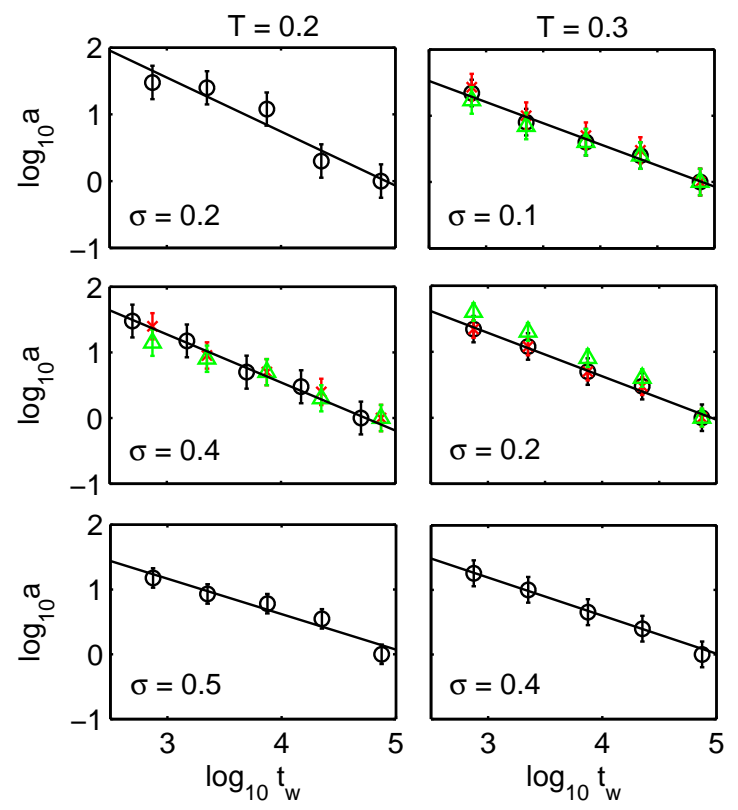

FIG. 3: Plot of the shift factors found by superimposing the creep compliance curves, $a_{J}$ (circles), the mean-squared displacement curves, $a_{M S D}$ (triangles), and the incoherent scattering function curves, $a_{C}(\times)$ at different wait times (see text). The solid lines are linear fits to the data.

the aging exponent [4], although it is not always the case that the aging process slows down with applied stress; stress has been known to increase the rate of aging in some circumstances as well [9, 10]. The structural origins of this effect are not well understood [11, 12].

At $T=0.3 u_{0} / k_{B}$, we find that the aging exponent is somewhat smaller than at $T=0.2 u_{0} / k_{B}$ and varies much less with applied stress. This behavior is most likely due to the fact that the temperature is approaching $T_{g}$. Indeed, experiments show that $\mu$ rapidly drops to zero above $T_{g}$. compliance is an order of magnitude larger at $T=0.3 u_{0} / k_{B}$ than at $T=0.2 u_{0} / k_{B}$ and the data does not fully superimpose in a master curve for long times where $J>0.2 u_{0} / a^{3}$. Shift factors were obtained from the small creep portion of the curves.

The relatively simple relationship between shift factors and wait time permits construction of an expression that describes the entire master curve in Fig. 2. For creep times that are short compared to the wait time - such that minimal physical aging occurs over the timescale of the experiment - experimental creep compliance curves can be fit to a stretched exponential (typical of processes with a spectrum of relaxation times),

$$
J(t)=J_{0} \exp \left[\left(t / t_{0}\right)^{m}\right]
$$

where $t_{0}$ is the retardation factor, and the exponent, $m$, has been found to be close to $1 / 3$ for most glasses [4]. A fit of this expression to our simulated creep compliance curves is shown in Fig. 2 (dotted line). This ex- 


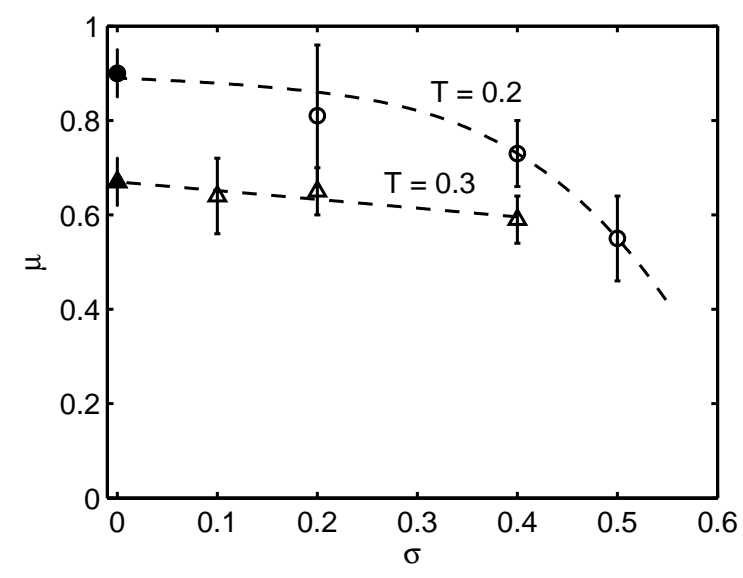

FIG. 4: The aging exponent, $\mu$, determined from the slopes of $\log \left(a_{J}\right)$ versus $\log \left(t_{w}\right)$ (from Fig. 3) plotted versus stress (open symbols). The solid symbols at zero stress refer to shift factors determined from $a_{M S D}$ (eq. 7) and $a_{C}$ (eq. 6) data only. The dashed lines are guides to the eye.

pression is clearly only consistent with the data at times $t<t_{w}$. At times much longer than the wait time, the creep compliance varies more slowly due to the stiffening caused by aging during the course of the experiment. Struik suggested that eq. (41) could be extended to the long-time creep regime, where the experimental timescale may be longer than the wait time, by introducing an effective time to account for the slowdown in the relaxation timescales:

$$
t_{\mathrm{eff}}=\int_{0}^{t}\left(\frac{t_{w}}{t_{w}+t^{\prime}}\right)^{\mu} d t^{\prime}
$$

Upon replacing $t$ with $t_{\text {eff }}$, eq. (4) may be used to describe the entire creep curve. Creep compliance curves from Fig. 2 can indeed be fit to this form (dashed lines) for a known wait time, $t_{w}$, and aging exponent, $\mu$, as obtained from the master curve. We find $m \approx 0.5 \pm 0.1$ for all stresses at $T=0.2 u_{0} / k_{B}$, and a relatively broad range of values for $J_{0}$ and $t_{0}$ are consistent with the data. For the simple thermo-mechanical history prescribed by the creep experiment, Struik's effective time formulation appears to work quite well.

The present results parallel those of a recent simulation study of the shear yield stress in glassy solids [21]. In this work, the glassy solid was deformed at constant strain rate, and two different regimes of strong and weak rate dependence emerged depending on the time to reach the yield point relative to the wait time. In order to rationalize these results, a rate-state model was developed that accounted for the internal evolution of the material with age through a single state variable $\Theta(t)$. This formulation successfully collapses yield stress data for different ages and strain rates in a universal curve by adapting the evolution of the state variable during the strain interval. We note here that this state variable is closely related to
Struik's effective time, as it tries to subsume the modified aging dynamics during deformation in a single variable and in particular easily includes the effects of overaging or rejuvenation.

\section{B. Microscopic Dynamics}

The aging behavior of the simulated mechanical response functions agrees remarkably well with experiment. Additional microscopic information from simulations allows us to obtain more directly the relevant timescales of the system, and the relevant microscopic processes responsible for aging. One parameter which has been useful in studying glassy dynamics is the "self" part of the incoherent scattering factor [19],

$$
C_{q}\left(t, t_{w}\right)=\frac{1}{N} \sum_{j=1}^{N} \exp \left(i \vec{q} \cdot\left[\vec{r}_{j}\left(t_{w}+t\right)-\vec{r}_{j}\left(t_{w}\right)\right]\right)
$$

where $\overrightarrow{r_{j}}$ is the position of the $j^{\text {th }}$ atom, and $\vec{q}$ is the wave-vector. $C_{q}$ curves as a function of age are shown in Fig. 5] and exhibit three distinct regions. Initially, $C_{q}$ decreases as particles make very small unconstrained excursions about their positions. There follows a long plateau, where the correlation function does not change considerably. In this regime, atoms are not free to diffuse, but are trapped in local cages formed by their nearest neighbours. For this reason, the time spent in the plateau regime is often associated with a "cage time". The plateau region ends when particles finally escape from local cages ( $\alpha$-relaxation), and larger atomic rearrangements begin to take place. The cage time corresponds closely to the transition from short-time to longtime regime observed in the creep compliance. Structural rearrangements taking place in the $\alpha$-relaxation regime are clearly associated with the continued aging observed in the creep compliance, as well as plastic deformations occurring in that region.

The correlation functions for different ages are similar in form, but the time spent in the plateau region increases with age. Just as creep compliance curves can be shifted in time to form a master curve, we may overlap the long-time, cage-escape regions of $C_{q}$ by rescaling the time variable of the correlation data at different ages (see inset of Fig. 5). The corresponding shift factors $a_{C}\left(t_{w}\right)$ are also shown in Fig. 3. where we see that the increase in cage time with age follows the same power law as the shift factors of the creep compliance. These results are qualitatively similar to the scaling of the relaxation times with age found in [19] with no load.

The real space quantity corresponding to $C_{q}$ is the mean squared displacement,

$$
\left\langle\vec{r}\left(t, t_{w}\right)^{2}\right\rangle=\frac{1}{N} \sum_{j=1}^{N} \Delta \vec{r}_{j}\left(t, t_{w}\right)^{2}
$$




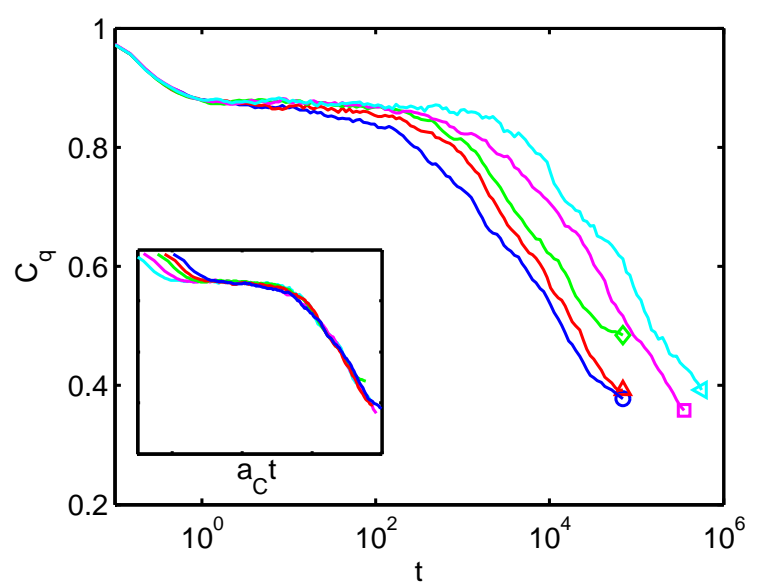

FIG. 5: Incoherent scattering factor (eq. 6) for different wait times measured under the same loading conditions as in Fig. 1(a) for $q=(0,0,2 \pi)$. The inset shows the master curve created by rescaling the time variable by $a_{C}$. Symbols as in Fig. 11(b).

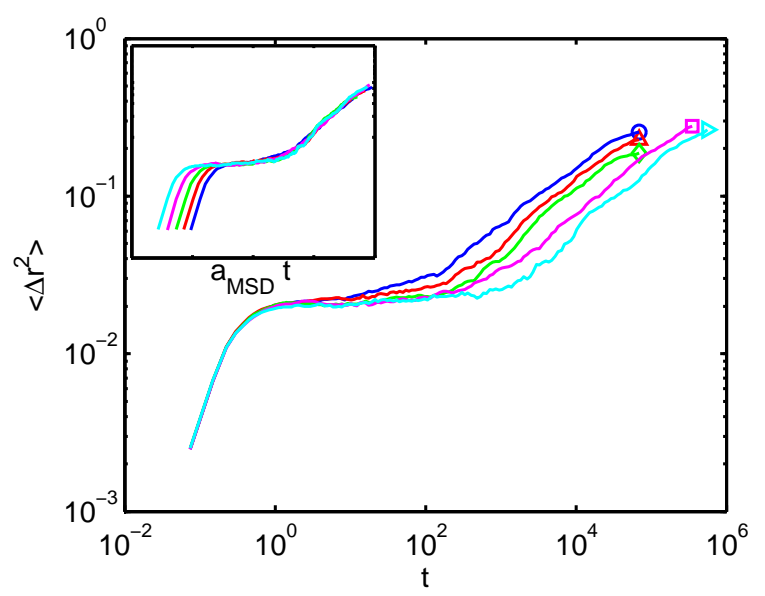

FIG. 6: Mean-squared displacement (eq. 7) for different wait times measured under the same loading conditions as in Fig. 1(a). The inset shows the master curve created by rescaling the time variable by $a_{M S D}$. Symbols as in Fig. 1(b).

where $\Delta \vec{r}_{j}\left(t, t_{w}\right)=\vec{r}_{j}\left(t_{w}+t\right)-\vec{r}_{j}\left(t_{w}\right)$. This function is shown in Fig. 6] Again we see three characteristic regions of unconstrained (ballistic), caged, and cage-escape behavior. The departure from the cage plateau likewise increases with age, and a master curve can be constructed by shifting the curves with a factor $a_{M S D}$ (see inset of Fig. 6). Shift factors $a_{M S D}$ are plotted in Fig. 3, along with shifts for creep compliance and incoherent scattering function. As anticipated, the shifts versus wait time for $\left\langle\Delta r^{2}\right\rangle$ fully agree with those obtained from $C_{q}$ and $J$. This clearly demonstrates that for our model, the cage escape time is indeed the controlling factor in the aging dynamics of the mechanical response functions.

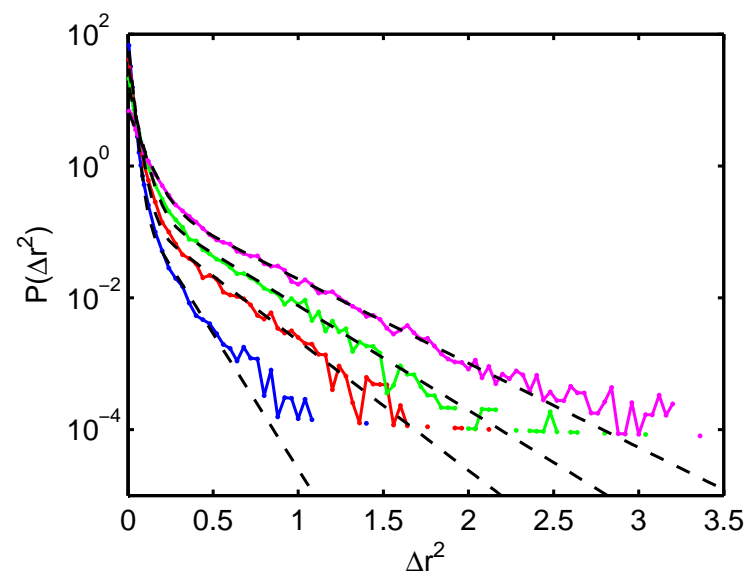

FIG. 7: The displacement probability distribution versus time measured under the same loading conditions as in Fig. 1(a), with a wait time of $500 \tau_{L J}$. The solid lines from left to right are obtained at times $t$ of $75,750,7500$, and $75000 \tau_{L J}$. The dashed lines show fits to the double Gaussian distribution (see text, eq. 8).

Additional information about microscopic processes can be obtained by studying not only the mean of the displacements, but also the full spectrum of relaxation dynamics as a function of time and wait time. To this end, we measure the probability distribution $P\left(\Delta r\left(t, t_{w}\right)^{2}\right)$ of atomic displacements during time intervals, $t$, for glasses at various ages, $t_{w}$. This quantity is complementary to the measurements of dynamical heterogeneities detailed in [26], where the spatial variations of the vibrational amplitudes were measured at a snapshot in time to show the correlations of mobile particles in space. In our study, we omit the spatial information, but retain all of the dynamical information.

Representative distribution functions are shown in Fig. 7 for a constant wait time of $t_{w}=500 \tau_{L J}$ and various time intervals $t$. The distributions were obtained from a smaller system of only 271 polymer chains due to memory constraints. The data does not reflect a simple Gaussian distribution, but clearly exhibits the presence of two distinct scales: there is a narrow distribution of caged particles and a wider distribution of particles that have escaped from their cages. This behavior can be described by the sum of two Gaussian peaks,

$$
P\left(\Delta r^{2}\right)=N_{1} \exp \left(\frac{-\Delta r^{2}}{\sigma_{1}^{2}}\right)+N_{2} \exp \left(\frac{-\Delta r^{2}}{\sigma_{2}^{2}}\right) .
$$

Deviations from purely Gaussian behavior are common in glassy systems and are a signature of dynamical heterogeneities [26, 27]. Experiments on colloidal glasses 28] show a similar separation of displacement distributions into fast and slow particles.

A fit of the normalized distributions to eq. (8) (dashed lines in Fig. 7) requires adjustment of three parameters: the variance of caged and mobile particle distributions, 

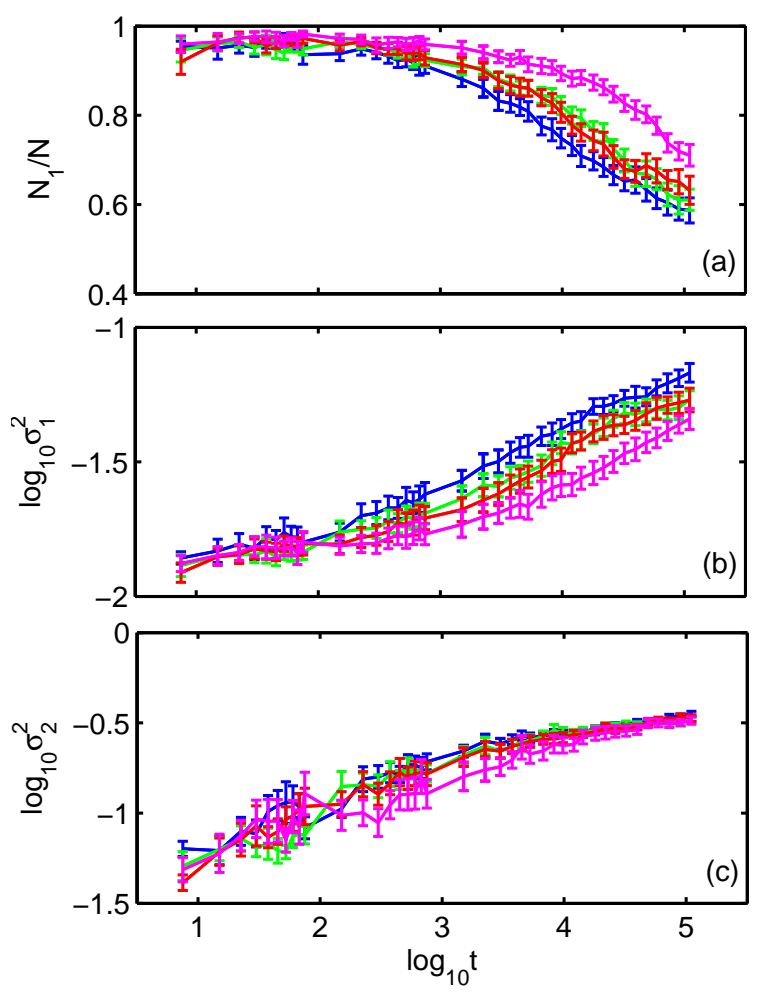

FIG. 8: The Gaussian fit parameters for the distribution of displacements (see text, eq. 8), (a) $N_{1} / N$, (b) $\sigma_{1}^{2}$, and (c) $\sigma_{2}^{2}$ measured under the same loading conditions as in Fig. 1(a). The curves are for wait times increasing from left to right from $500 \tau_{L J}$ to $15000 \tau_{L J}$.

$\sigma_{1}^{2}$ and $\sigma_{2}^{2}$, as well as their relative contributions $N_{1} / N$, where $N=N_{1}+N_{2}$. These parameters are sufficient to describe the full evolution of the displacement distribution during aging. In Fig. 8 we show the fit parameters as a function of time and wait time. Again two distinct time scales are evident. At short times, most of the particles are caged $\left(N_{1} / N \approx 1\right)$, and the variance of the cage peak is also changing very little. There are few rearrangements in this regime, however Fig. 8(c) shows that a small fraction of particles are mobile at even the shortest times. At a time corresponding to the onset of cage escape, the number of particles in the cage peak begins to rapidly decay, and the variance of the cage peak increases. This indicates that the cage has become more malleable - small, persistent rearrangements occur leading to eventual cage escape. In this regime, the variance of the mobile peak increases very little. Note that the typical length scale of rearrangements is less than a particle diameter even in the cage escape regime, but the number of particles undergoing rearrangements changes by more than $50 \%$.

Similar to the compliance and mean-squared displacement curves, the data in Fig. 8 (a) and (b) can also be

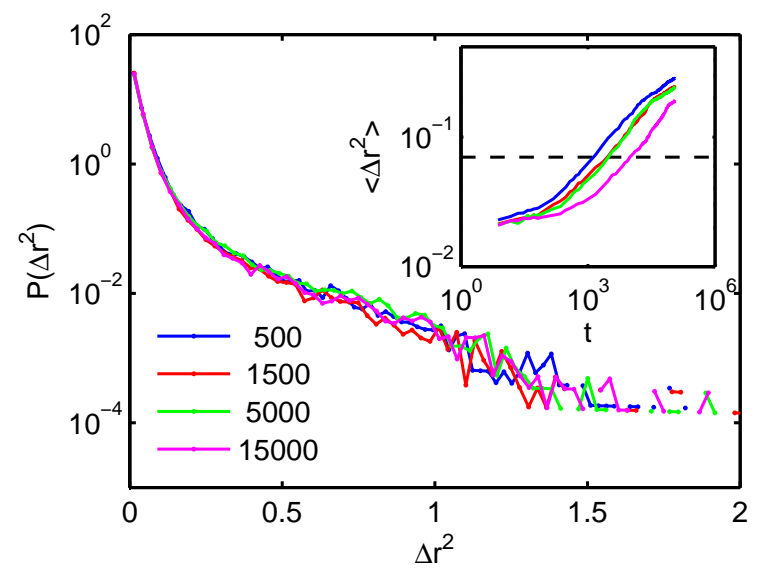

FIG. 9: The displacement probability distribution measured under the same loading conditions and wait times as in Fig. 1(a) plotted at times corresponding to $\left\langle r^{2}\left(t, t_{w}\right)\right\rangle=$ 0.7 , shown in the inset as a dashed line. The legend indicates the wait time.

superimposed by shifting time. Shift factors for $N_{1} / N$ and $\sigma_{1}^{2}$ coincide exactly with shifts for the mean; however, data for $\sigma_{2}^{2}$ (Fig. 8(c)) seems to be much less affected by the wait time. The aging dynamics appears to be entirely determined by the cage dynamics, and not by larger rearrangements within the glass.

Since the fit parameters exhibit the same scaling with wait time as the mean, one might expect that the entire distribution of displacements under finite load scales with the evolution of the mean. In Fig. 9, we plot displacement distributions for several wait times at time intervals chosen such that the mean squared displacements are identical (see inset). Indeed, we find that all curves overlap, indicating that the entire relaxation spectrum ages in the same way. A similar observation was recently made in simulations of a model for a metallic glass aging at zero stress [29], although in this study the tails of the distribution were better described by stretched exponentials.

In order to study the effect of load on the relaxation dynamics, we compare in Figure10 the fit parameters for a sample undergoing creep (replotted from Fig. 8) and a reference sample without load. It is clear that the dynamics are strongly affected by the applied stress only in the region characterized by $\alpha$-relaxations. For the stress applied here, the onset of cage-escape does not appear to be greatly modified by the stress, however the decay in $N_{1} / N$ and the widening of the cage peak are accelerated. The stress does not modify the variance of the mobile peak, confirming again the importance of local rearrangements as compared to large-scale motion in the dynamics of the system. The accelerated structural rearrangements caused by the stress result in creep on the macroscopic scale, but may also be responsible for the modification of the aging dynamics with stress as observed in Fig. 4 

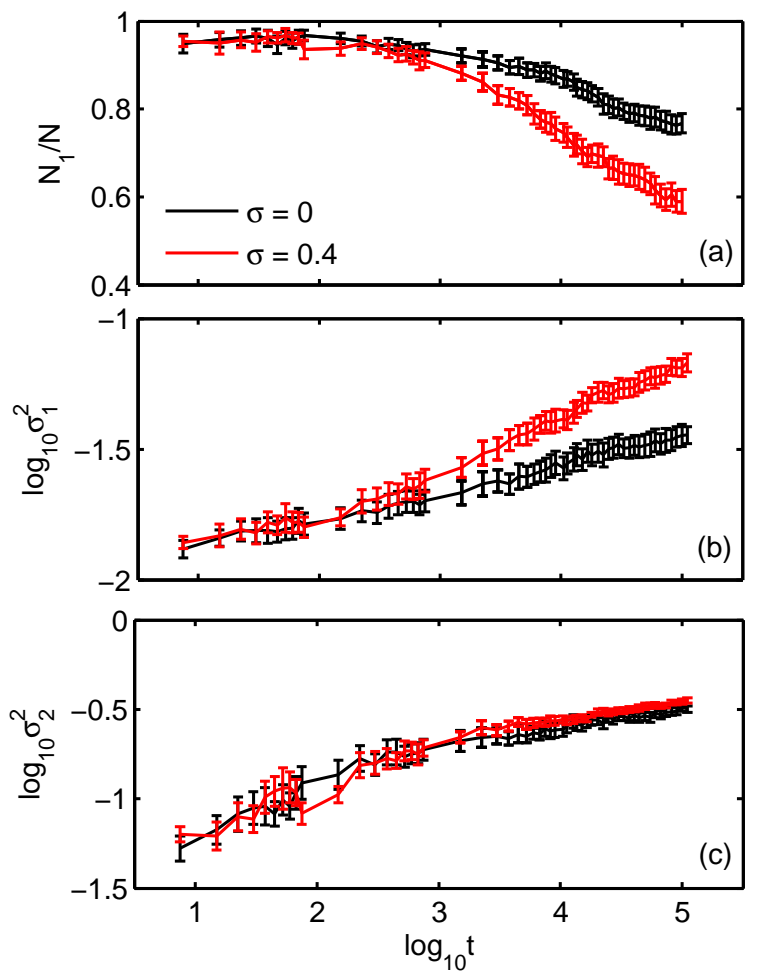

FIG. 10: The Gaussian fit parameters to the displacement distributions (see text, eq. 8) (a) $N_{1} / N$, (b) $\sigma_{1}^{2}$, and (c) $\sigma_{2}^{2}$ of a sample aged at $T=0.2 u_{0} / k_{B}$ for $t_{w}=500 \tau_{L J}$, and then either undergoing a creep experiment at $\sigma=0.4 u_{0} / a^{3}$ (black), or simply aging further at zero stress (red).

\section{Structural evolution}

The connection between the dynamics and the structure of a glass during aging remains uncertain, mostly because no structural parameter has been found that strongly depends on wait time. Recent simulation studies of metallic glasses have shown the existence of several short range order parameters that can distinguish between glassy states created through different quenching protocols [30, 31, 32]. A strong correlation has been found between "ordered" regions of the glass and strain localization. Many metallic glasses are known to form quasi-crystalline structures that optimize local packing. It remains to be seen whether the short-range order evolves in the context of aging and in other glass formers such as polymers and colloids. A recent experimental study of aging in colloidal glasses found no change in the distribution function of a tetrahedral order parameter 33]. Below, we investigate several measures of local order in our model as they evolve with age and under load.

Since Lennard-Jones liquids are known to condense into a crystal with fcc symmetry at low temperatures, it is reasonable to look for the degree of local fcc order in our polymer model. The level of fcc order can be quantified via the bond orientational parameter [34],

$$
Q_{6}=\left(\frac{4 \pi}{13} \sum_{m=-6}^{6}\left|\overline{Y_{6 m}}\right|^{2}\right)^{1 / 2}
$$

This parameter has been successfully used to characterize the degree of order in systems of hard sphere glasses. $Q_{6}$ is determined for each atom by projecting the bond angles of the nearest neighbours onto the spherical harmonics, $Y_{6 m}(\theta, \phi)$. The overbar denotes an average over all bonds. Nearest neighbours are defined as all atoms within a cutoff radius, $r_{c}$, of the central atom. For all of the order parameters discussed here, the cutoff radius is defined by the first minimum in the pair correlation function, in this case $1.45 a$. $Q_{6}$ is approximately 0.575 for a perfect fcc crystal; for jammed structures, it can exhibit a large range of values less than about 0.37 [34]. The full distribution of $Q_{6}$ for our model glass is shown for several ages as well as an initial melt state in Fig. 11(a). We see that there is very little difference even between melt and glassy states in our model, and no discernible difference at all with increasing age.

Locally, close-packing is achieved by tetrahedral ordering and not fcc ordering, however, tetrahedral orderings cannot span the system. The glass formation process has been described in terms of frustration between optimal local and global close-packing structures. To investigate the type of local ordering in this model, we investigate a 3-body angular correlation function, $P(\theta)$. $\theta$ is defined as the internal angle created by a central atom and individual pairs of nearest-neighbours, and $P(\theta)$ is the probability of occurrence of $\theta$. Results for this correlation are shown in Fig. 11(b). Two peaks at approximately $60^{\circ}$ and $110^{\circ}$ indicate tetrahedral ordering. The peaks sharpen under quenching from the melt, but the distribution does not evolve significantly during aging. In contrast, simulations of metallic glass formers showed a stronger sensitivity of this parameter to the quench protocol [31], but most of those changes may be due to rearrangements in the supercooled liquid state and not in the glassy state.

Another parameter that has been successful in classifying glasses is the triangulated surface order parameter [32],

$$
S=\sum_{q}(6-q) \nu_{q}
$$

which measures the degree of quasi-crystalline order. The surface coordination number, $q$, is defined for each atom of the coordination shell as the number of neighbouring atoms also residing in the coordination shell; $\nu_{q}$ is the number of atoms in the coordination shell with surface coordination $q$. Ordered systems have been identified with $S$ equal to 12 (icosahedron), 14, 15 and 16. Figure 111(c) shows the probability distribution for $P(S)$ for the melt and for glassy states with short and long wait times. 

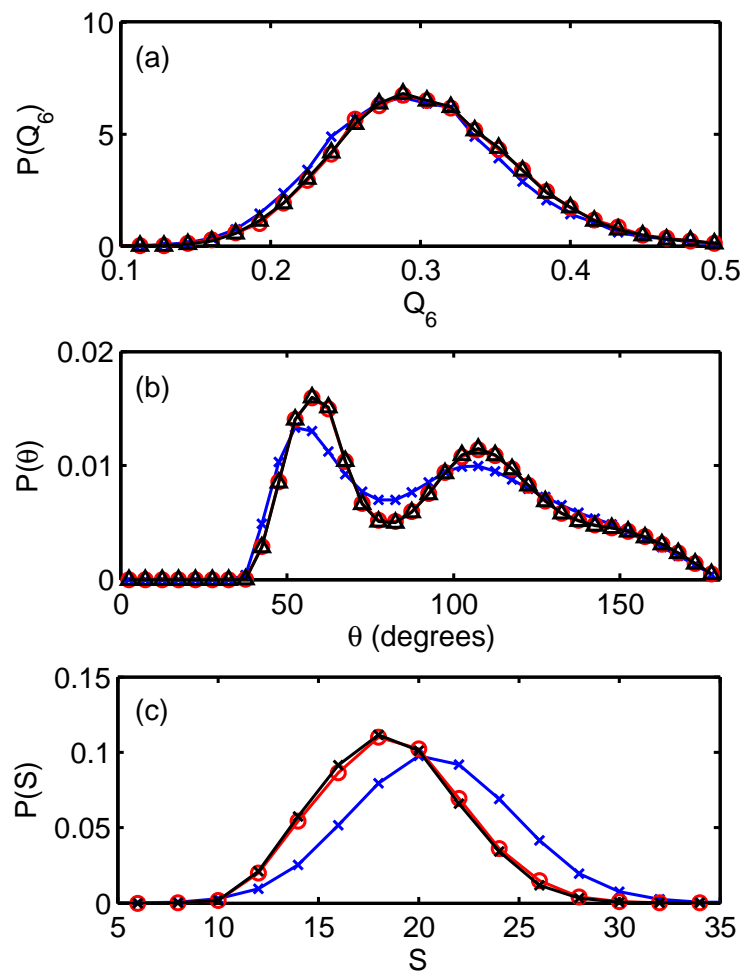

FIG. 11: Short-range order parameters: (a) the bondorientational parameter, (b) the three-body angular correlations, (c) the surface triangulated order (see text for discussion). x's show the melt state, circles show the sample aged for $t_{w}=500 \tau_{L J}$, and triangles show the sample aged for $500,000 \tau_{L J}$.

The peak of the distribution moves toward lower $S$ (more ordered) upon cooling, and continues to evolve slowly in the glass. The mean of $S$ relative to the as-quenched state, $\langle S\rangle$, is shown in Fig. 12 as a function of wait time at two temperatures. We see that $\langle S\rangle$ is a logarithmically decreasing function of wait time. Even though this is not a strong dependence, this order parameter is significantly more sensitive to age than the others that have been investigated.

Figure 12 also shows the order parameter $\langle S\rangle$ after the ramped-up stress has been applied to the aged samples. We can see that at $T=0.2 u_{0} / k_{B}$, some of the order that developed during age is erased, while no appreciable change occurs at the higher temperature $T=0.3 u_{0} / k_{B}$. These results correlate well with the behavior of the aging exponent found in Fig. 4, where mechanical rejuvenation was found at lower temperatures but was much less pronounced at higher $T$. The load is applied very quickly, and most of the deformation in this regime is affine, however, the strain during this time was similar for both temperatures, therefore the effect is not simply due to a change in density. More work is needed to clarify the nature of the structural changes during rejuvenation.
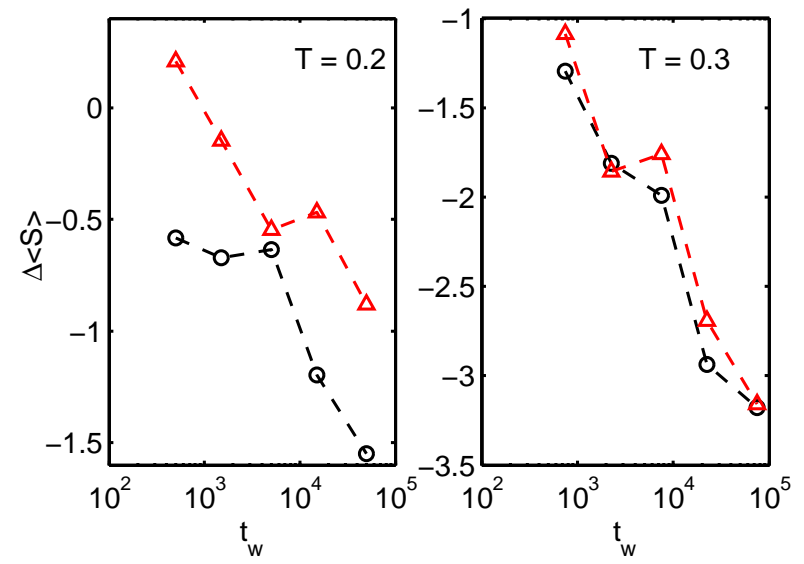

FIG. 12: Precent change in the triangulated surface order parameter with wait time as compared to the just-quenched sample. Circles are for samples aged at zero pressure for the time $t_{w}$. Triangles are for the same samples immediately after ramping up to the creep stress. For $T=0.2 u_{0} / k_{B}$ this stress is $0.4 u_{0} / a^{3}$, and for $T=0.3 u_{0} / k_{B}$ the stress is $0.1 u_{0} / a^{3}$.

\section{CONCLUSIONS}

We investigate the effects of aging on both macroscopic creep response and underlying microscopic structure and dynamics through simulations on a simple model polymer glass. The model qualitatively reproduces key experimental trends in the mechanical behavior of glasses under sustained stress. We observe a power-law dependence of the relaxation time on the wait time with an aging exponent of approximately unity, and a decrease in the aging exponent with increasing load that indicates the presence of mechanical rejuvenation. The model creep compliance curves can be fit in the short and long-time regimes using Struik's effective time formulation. Additionally, investigation of the microscopic dynamics through two-time correlation functions has shown that, for our model glass, the aging dynamics of the creep compliance exactly corresponds to the increase in the cage escape or $\alpha$-relaxation time.

A detailed study of the entire distribution of particle displacements yields an interesting picture of the microscopic dynamics during aging. The distribution can be described by the sum of two Gaussians, reflecting the presence of caged and mobile particles. The fraction of mobile particles and the amplitude of rearrangements in the cage strongly increase at the cage escape time. However, in analogy with results in colloidal glasses [35], structural rearrangements occur even for times well within the "caged" regime, and fairly independent of wait time and stress. For our model glass, we find that the entire distribution of displacements scales with age in 
the same way as the mean. At times when the longtime portion of the mean squared displacement overlaps, the distribution of displacements at different wait times completely superimpose, confirming that all of the mechanical relaxation times scale in the same way with age.

To characterize the evolution of the structure during aging, we investigate several measures of short-range order in our model glass. We find that the short-range order does not evolve strongly during aging. The triangulated surface order [32], however, shows a weak logarithmic dependence on age. Results also show a change in structure when a load is rapidly applied, and this seems to be correlated with an observed decrease in the aging exponent under stress.

This study has characterized the dynamics of a model glass prepared by a rapid quench below $T_{g}$, followed by aging at constant $T$ and subsequent application of a constant load. For such simple thermo-mechanical histories, existing phenomenological models work well, however, the dynamics of glasses are in general much more complex. For instance, large stresses in the non-linear regime modify the aging dynamics and cause nontrivial effects such as mechanical rejuvenation and over-aging [10, 11]. Also, experiments have shown that the time-age time superposition no longer holds when polymer glasses undergo more complex thermal histories such as a temperature jump [23]. The success of our study in analyzing simple aging situations indicates that the present simulation methodology will be able to shed more light on these topics in the near future.

\section{Acknowledgments}

We thank the Natural Sciences and Engineering Council of Canada (NSERC) for financial support. Computing time was provided by WestGrid. Simulations were performed with the LAMMPS molecular dynamics package [36].
[1] J.-L. Barrat, M. V. Feigelman, J. Kurchan, and J. D. (Eds.), Slow Relaxations and Nonequilibrium Dynamics in Condensed Matter: Les Houches Session LXXVII (Springer, 2002).

[2] C. A. Angell, Science 267, 1924 (1995).

[3] C. A. Angell, K. L. Ngai, G. B. McKenna, P. F. McMillan, and S. W. Martin, J. Applied Physics 88, 3113 (2000).

[4] L. C. E. Struik, Physical Aging in Amorphous Polymers and Other Materials (Elsevier, Amsterdam, 1978).

[5] J. M. Hutchinson, Prog. Polym. Sci. 20, 703 (1978).

[6] B. Abou, D. Bonn, and J. Meunier, Phys. Rev. E 64, 021510 (2001).

[7] M. Cloitre, R. Borrega, and L. Leibler, Phys. Rev. Lett. 85, 4819 (2000).

[8] T. Jonsson, J. Mattsson, C. Djurberg, F. A. Khan, P. Nordblad, and P. Svedlindh, Phys. Rev. Lett. 75, 4138 (1995).

[9] V. Viasnoff and F. Lequeux, Phys. Rev. Lett. 89, 065701 (2002).

[10] D. J. Lacks and M. J. Osborne, Phys. Rev. Lett. 93, 255501 (2004).

[11] G. B. McKenna, J. Phys.: Condens. Matter 15, S737 (2003).

[12] L. C. E. Struik, Polymer 38, 4053 (1997).

[13] M. Utz, P. G. Debenedetti, and F. H. Stillinger, Phys. Rev. Lett. 84, 1471 (2000).

[14] C. Monthus and J. P. Bouchaud, J. Phys. A: Math. Gen. 29, 3847 (1996).

[15] P. Sollich, F. Lequeux, P. Hebraud, and M. E. Cates, Phys. Rev. Lett. 78, 2020 (1997).

[16] K. Chen and K. S. Schweizer (2007), preprint, personal communication.

[17] W. Kob, J. Phys.: Condens. Mat. 11, R85 (1999).

[18] C. Bennemann, W. Paul, K. Binder, and B. Duenweg, Phys. Rev. E 57, 843 (1998).

[19] W. Kob and J.-L. Barrat, Phys. Rev. Lett. 78, 4581
(1997).

[20] F. Varnik, L. Bocquet, and J.-L. Barrat, J. Chem. Phys 120, 2788 (2004).

[21] J. Rottler and M. O. Robbins, Phys. Rev. Lett. 95, 225504 (2005).

[22] K. Kremer and G. S. Grest, J. Chem. Phys 92, 5057 (1990).

[23] H. Montes, V. Viasnoff, S. Juring, and F. Lequeux, J. Stat. Mech.: Theory and Experiment p. P03003 (2006).

[24] L. C. Brinson and T. S. Gates, Int. J. Solids and Structures 32, 827 (1995).

[25] G. B. McKenna and A. J. Kovacs, Polym. Eng. Sci. 24, 1131 (1984).

[26] K. Vollmayr-Lee and A. Zippelius, Phys. Rev. E 72, 041507 (2005).

[27] W. Kob, C. Donati, S. J. Plimpton, P. H. Poole, and S. C. Glotzer, Phys. Rev. Lett. 79, 2827 (1997).

[28] E. R. Weeks and D. A. Weitz, Chem. Phys. 284, 361 (2002).

[29] H. E. Castillo and A. Parsaeian, Nature Physics 3, 26 (2007).

[30] Y. Shi and M. L. Falk, Phys. Rev. Lett. 95, 095502 (2005).

[31] F. Albano and M. L. Falk, J. Chem. Phys 122, 154508 (2005).

[32] Y. Shi and M. L. Falk, Phys. Rev. B 73, 214201 (2006).

[33] G. C. Cianci, R. E. Courtland, and E. R. Weeks, in Flow Dynamics: The Second International Conference on Flow Dynamics (AIP Conf. Proc., 2006), vol. 832, pp. $21-25$.

[34] S. Torquato, T. M. Truskett, and P. G. Debenedetti, Phys. Rev. Lett. 84, 2064 (2000).

[35] R. E. Courtland and E. R. Weeks, J. Phys.:Condens. Matter 15, S359 (2003).

[36] http://lammps.sandia.gov. 